\title{
Correlates to Relapse as Perceived by Schizophrenic Patients
}

\author{
Zeinab Ahmed Abdelsalam, Naglaa Mostafa Gaber \\ Psychiatric Mental Health Nursing Department, Faculty of Nursing, Cairo University, Cairo, Egypt
}

Email address:

abdelsalam_zizi@yahoo.com (Z. A. Abdelsalam),naglaamostafa45@gmail.com (N. M. Gaber)

\section{To cite this article:}

Zeinab Ahmed Abdelsalam, Naglaa Mostafa Gaber. Correlates to Relapse as Perceived by Schizophrenic Patients. American Journal of Nursing Science. Vol. 6, No. 4, 2017, pp. 308-314. doi: 10.11648/j.ajns.20170604.15

Received: May 21, 2017; Accepted: May 31, 2017; Published: July 10, 2017

\begin{abstract}
Relapse among schizophrenic patients is a major challenge for mental health service providers in Egypt. Relapse has negative consequences and it can affect to patients, their families, the mental health care providers and the country's economy. This study aimed to explore the correlates of relapse as perceived by schizophrenic patients. A descriptive correlational design was utilized in this study. Three tools were utilized; the socio-demographic data sheet, the patient's medical data sheet and the relapse factors questionnaire that was developed by the researchers. A purposive sample of 90 schizophrenic patients was recruited in the current study. The study was carried out at Psychiatric Medicine and Addiction Prevention Hospital - El Manial University Hospital. The results revealed that the majority of the sample agreed that the social stigma related factors, and lack of rehabilitation services in the community, in addition to decreased patient's compliance with the medications were the most reported factors for relapse. However, the multiple regression analysis showed that there were no significant relationships among patient's socio-demographic or medical data and their relapse. The study concluded that, many factors can interfere with the recovery process of schizophrenic patients such as, lack of social and community support, non compliance to medication, and stigma. The study recommended that applying the mental health education and provision of effective and accessible community mental health services can help in reducing the relapse among schizophrenic patients.
\end{abstract}

Keywords: Correlates to Relapse, Schizophrenic Patients, Patient's Perspective

\section{Introduction}

Schizophrenia is a constant extreme issue described by wrecking signs and side effects, for example, visualizations, hallucinations, disarranged correspondence, and lack of common sense, lessened inspiration, and blunted influence [1]. Relapse has been accounted to happen with schizophrenia even while on treatment [2]. "Relapse has been characterized as an intensifying of psychopathological manifestations or re-hospitalization in the year after hospital release" [3]. Schizophrenia takes after a variable course, with finish symptomatic and social recuperation in around $33 \%$ of cases. Schizophrenia can however take after an incessant or intermittent course, with leftover manifestations and deficient social recuperation. People with chronic schizophrenia constituted an expansive extent of all occupants of mental establishments in the past and still do where these organizations exist [4].

Research studies done by W.H.O in a few nations have demonstrated that individuals can recuperate from mental issue like schizophrenia [3]. The course of schizophrenia is said to be inconsistent, it has a tendency to go into incessant stage with relapse and dispatching design. Studies have demonstrates that around $20 \%$ of schizophrenic patients have a solitary lifetime scene with further relapse [4].

Regardless of the likelihood of recovery, there are a few patients who continue going to the doctor's facility in light of a relapse. In a study that was done to track relapse in patients with schizophrenia it was discovered that $25 \%$ of subjects were admitted to intense care within three months of their last admission [5]. In another study on the cost of relapse and the indicators of relapse in the treatment of schizophrenia, the outcomes demonstrated that $20 \%$ of the members relapsed in the period of six months and it was additionally discovered that the cost for patients with earlier relapse was around three times the cost of patients without earlier relapse. This is connected with high psychiatric hospitalization with higher expenses of inpatient administrations and in addition 
outpatient benefits that incorporate prescription and individual treatment administrations [6].

Many relapses describe the course of disease in many patients with schizophrenia, yet the way of these episodes has not been broadly inquired about and clinicians may not generally know about vital ramifications.

\subsection{Significance of the Study}

The Egyptian literature has very few studies in respect to relapse and schizophrenia. None of these studies have investigated the patient's own perspective on the factors that may cause the relapse. Relapse in schizophrenia has a great deal of impacts to patients, caregivers, the wellbeing sector and the nation's economy.

Relapse can devastate the patient's ability to work productively and or even, the patient's motivation to find work; subsequently that can increase the financial burdens encountered by the patients and their families. The patient's family is the one who always pay for the patient's bills in the hospital once readmitted which turns out to be expensive. The health division is forced with an extensive raising issues related to the higher number of patients' re-hospitalization.

The effectiveness of the psychiatric nursing prevention starts from the nurse; who is caring most of the time of the psychiatric patient; and it is important to add to the nurses' knowledge the patient's perspective about the possible factors that may lead to relapse. The results of this study will help the psychiatric nurse to plan and implement an individualized nursing care when discharging the patient from the hospital while; incorporating the available community resources.

Additionally, this study will add to the body of nursing knowledge about the Egyptian schizophrenic patients' perspective of the relapse related factors. The study will shade the light on a combination of various factors such as the patients' socio-demographic and medical data and how they are associated with the patient's relapse.

\subsection{Aim of the Study}

The purpose of this study was to explore the correlates to relapse as perceived by schizophrenic patients.

\subsection{Research Questions}

Q1-What are the correlates to relapse among schizophrenic patients from their perspectives?

Q2- What are the relationships among patients' sociodemographic, medical data and their relapse?

\section{Subjects and Methods}

\subsection{Research Design}

A descriptive correlational design was utilized in this study. This type of research design is appropriate to the current study as the researches collected the data from the subjects at one time meeting without changing any of the subjects' behaviors or perception [7].

\subsection{Sample}

A purposive sample of 90 schizophrenic patients was recruited in the current study. The sample size for the study was determined by assuming relapse rate of $20 \%$ giving any particular outcome to be within $5 \%$ marginal error and $95 \%$ confidence interval of certainty (alpha $=0.05$ ). The researchers used (http://www.sample-size.net/sample-sizeconf-interval-proportion/) to calculate the sample size. Based on this assumption, the actual sample size calculated for this study was 90 subjects. The subjects of the current study were chosen according to the following inclusion criteria: 1) patients of schizophrenia aged between 18 and 65 years, b) patients who were able to understand the study questionnaire. The exclusion criteria included a) patients who were having organic mental disorder or chronic medical illness.

\subsection{Setting}

The study was carried out at Psychiatric Medicine and Addiction Prevention Hospital - El Manial University Hospital Out-Patient Department (OPD) in El Manial University Hospitals- Cairo University.

\subsection{Tools}

Two tools were utilized in the this study

1- Socio-demographic data sheet and medical data questionnaire - This included age, gender, marital status, education, monthly income of the patient, patient's diagnosis, duration of illness, number of previous admission, type of medications, number of follow-up in the last month, date of last admission, date of last discharge.

2- Relapse questionnaire. A specially designed semistructured questionnaire adopted from Chabungbam, Avasthi, and Sharan [8] and was used to elicit the factors which responsible for relapse and remission in mentally ill patients. It consists of five sub-scales which contains twenty five questions. Sub-scale 1) individual factors of the patient; sub-scale 2) social factors; sub-scale 3) societal stigma; subscale 4) rehabilitation of patient in the community; and subscale 5) compliance with the medications. Each sub-scale contains five questions, the questionnaire was rated on three point Likert scale in which (1)= Agree, (2)= Uncertain, (3)= Disagree. The higher scores indicate higher relapse rate. The reliability of the questionnaire was tested in this study and it was (.79) which is considered good level of reliability. The validation of questionnaire was done by submitting it to a panel of five experts; four of them were in psychiatric mental health nursing specialty and one statistician.

\subsection{Procedure}

After obtaining the official approval from the director of Psychiatric Medicine and Addiction Prevention Hospital - El Manial University Hospital, the mental health nurse incharge of the Out-patient department was informed about the aim and procedures of the study and asked to help in identifying subjects for the in-depth interviews. Purposive 
sampling strategy was employed. In this case the researcher deliberately chose the cases that could best contribute to the information needs of the study [7]. An individualized interview was done for each subject who met the inclusion criteria for about 25 to 30 minutes.

\subsection{Pilot Study}

The questionnaire was pretested on a sample equal to $10 \%$ of the total sample size that were not part of the main study. No further medications were done to the scale.

\subsection{Ethical Considerations}

The patients who met the inclusion criteria and agreed to participate in the current study were asked to an oral consent to agree in participating in the current study. Confidentiality and anonymity of the patients' information and freedom to withdraw at anytime during the study were ensured.

\subsection{Statistical Design}

Statistical analysis was done with the help of software 'SPSS 22' Statistical Package for the Social Sciences (Windows version 22.0). Descriptive statistics including number and percentages were used for qualitative variables and mean and standard deviations were used for quantitative data. Correlation coefficient and multiple regressions were used to answer the current research questions. Relationship between different measures was computed via Pearson's correlation coefficient. The level of significance in this study was $(<0.05)$, and $(<0.01)$ considered highly significant.

\section{Results and Data Analysis}

The results show that $(90 \%)$ of the studied schizophrenic patients were males and (40\%) their age ranged between 25 to 35 years. More than half of the studied schizophrenic patients $(54.4 \%)$ and $(53.3 \%)$ had secondary education and were single, respectively table 1 .

Table 2 illustrates that (50\%) had not enough income, and their income sources were $(36.7 \%)$ from free job, and (16.7\%) from working as employee, while; $(40 \%)$ had no source of income.

Table 1. Socio-demographic characteristics of the studied schizophrenic patients $(n=90)$.

\begin{tabular}{lll}
\hline Variable & $\mathbf{N}$ & $\mathbf{\%}$ \\
\hline Gender & & \\
Male & 81 & 90 \\
Female & 9 & 10 \\
Age & & \\
$<25$ years & 22 & 24.4 \\
25 - years & 36 & 40 \\
36- years & 14 & 15.6 \\
$>45$ years & 18 & 20 \\
Residence & & \\
Rural & 29 & 32 \\
Urban & 61 & 68 \\
Education & & \\
\hline
\end{tabular}

\begin{tabular}{lll}
\hline Variable & $\mathbf{N}$ & $\mathbf{\%}$ \\
\hline Higher education & 17 & 18.9 \\
Secondary & 59 & 65.5 \\
Education & 13 & 14.5 \\
Can read and write & 1 & 1.1 \\
Can't read or write & & \\
Marital status & 48 & 53.3 \\
Single & 37 & 41.1 \\
Married & 2 & 2.2 \\
Widow & 3 & 3.3 \\
Divorced &
\end{tabular}

Table 2. Frequency distribution of the studied schizophrenic patients according to their income and income resources $(n=90)$.

\begin{tabular}{lll}
\hline Variable & $\mathbf{N}$ & $\mathbf{\%}$ \\
\hline Income & & \\
Enough & 45 & 50 \\
Not enough & 45 & 50 \\
Income sources (non-exclusive answers) & & \\
Employee & 15 & 16.7 \\
Free job & 33 & 36.7 \\
Subsidy & 2 & 2.2 \\
Retirement & 3 & 3.3 \\
Relatives & 1 & 1.1 \\
None & 36 & 40 \\
\hline
\end{tabular}

Table 3 indicates that almost half of the studied schizophrenic patients (46.7\%) had one of their parents accompanied them during their psychiatrist visits, while; (4.4\%) had their offspring gone with them during the followup. Almost three quarters (72\%) had their illness for less than five years, and slightly more than three quarters (79.8\%) were admitted more than five times and $(85.7 \%)$ follow for less than three times.

Table 3. Clinical characteristics of the studied schizophrenic patients $(n=90)$.

\begin{tabular}{lll}
\hline Variable & $\mathbf{N}$ & $\mathbf{\%}$ \\
\hline $\begin{array}{l}\text { Relatives accompanied the patient (non- } \\
\text { exclusive answers) }\end{array}$ & \\
One of the parents & 44 & 46.7 \\
Siblings & 28 & 30 \\
Spouses & 16 & 16.7 \\
Offspring & 4 & 4.5 \\
Duration of illness & & \\
$<5$ years & 65 & 72 \\
$>5$ years & 25 & 28 \\
Number of Admission & & \\
$<3$ times & 71 & 79.8 \\
$>3$ times & 19 & 21.2 \\
Number of Follow-up & & \\
$<3$ times & 77 & 85.5 \\
$>3$ times & 13 & 14.4 \\
\hline
\end{tabular}

Table 4 reveals that $(87 \%, 71 \%, 71.15$, and $70 \%)$ of the studied schizophrenic patients respectively reported that they disagree that, no work skills, inability to reason well, lack good manner when talking to others and do not communicate effectively are the main personal factor of relapse. However, (24.4\%, and $24.4 \%$ ) of the studied sample reported that they are agree that communication skills, and misunderstanding the purpose of the medication were the main reasons of relapse. 
Table 4. Personal factors of relapse as reported by the studied schizophrenic patients $(n=90)$.

\begin{tabular}{llll}
\hline \multirow{2}{*}{ Personal factors } & Disagree & Uncertain & Agree \\
\cline { 2 - 4 } & $\mathbf{N}(\%)$ & $\boldsymbol{N}(\%)$ & $\boldsymbol{N}(\%)$ \\
\hline Schizophrenia patients do not have communication skills, i.e. do not communicate effectively & $63(70)$ & $5(5.6)$ & $22(24.4)$ \\
Schizophrenia patients have no work skills, for example, cannot wash themselves & $70(78.7)$ & $7(7.9)$ & $12(13.4)$ \\
Schizophrenia patients do not reason well & $64(71.1)$ & $12(13.3)$ & $14(15.5)$ \\
He/she has a lack of good manner of approach when talking to others & $64(71.1)$ & $9(10.0)$ & $17(18.8)$ \\
He/she cannot understand medicines when going to the hospital alone & $58(64.4)$ & $10(11.1)$ & $22(24.5)$ \\
\hline
\end{tabular}

Table 5 reveals that $(70 \%$, and $62.2 \%)$ of the studied schizophrenic patients respectively reported that they disagreed that they should be left alone because s/he will hurt her/himself and allocated tasks to perform are the main social factors of relapse. However, (47.8\% and 43.3\%) respectively reported that they agree that difficulty to go to clinic for consultation and costs too much to visit hospitals are the main social factors of relapse.

Table 5. Social factors of relapse as reported by the studied schizophrenic patients $(n=90)$.

\begin{tabular}{|c|c|c|c|}
\hline \multirow{2}{*}{ Social factors } & Disagree & Uncertain & Agree \\
\hline & $N(\%)$ & $N(\%)$ & $N(\%)$ \\
\hline I think schizophrenic patient should be allocated tasks to perform at home & $56(62.3)$ & $16(17.8)$ & $18(20)$ \\
\hline I think he/she should not be left alone because he/she will hurt him-/herself & $63(70)$ & $21(23.3)$ & $6(6.7)$ \\
\hline I think he should be reminded him/her about follow up dates & $36(40)$ & $21(23.3)$ & $33(36.6)$ \\
\hline It is difficult to go to the clinic for consultation because of distance & $31(34.4)$ & $16(17.8)$ & $43(47.8)$ \\
\hline It costs too much to visit the hospital & $30(33.3)$ & $21(23.3)$ & $39(43.3)$ \\
\hline
\end{tabular}

Regarding the social stigma factors, table 6 revealed that, $(48.9 \%$ and $46.8 \%)$ of the studied schizophrenic patients reported that they disagree that community members do not want to associate with schizophrenic patients and it is difficult to relate to schizophrenic patients are the main social stigma factors of relapse. However, $(65.5 \%, 47.7 \%$, and $42.2 \%)$ of the studied schizophrenic patients respectively agreed with respect schizophrenic patients, hospitalization is better, and difficult to relate to schizophrenic patients are the main social stigma factors of relapse.

Table 6. Social stigma factors as reported by the studied schizophrenic patients $(n=90)$.

\begin{tabular}{|c|c|c|c|}
\hline \multirow{2}{*}{ Social Stigma factors } & D & $\mathbf{U}$ & A \\
\hline & $N(\%)$ & $N(\%)$ & $N(\%)$ \\
\hline It is difficult to be related to a schizophrenic patient & $42(46.8)$ & $10(11.1)$ & $38(42.2)$ \\
\hline It is better to hospitalize him/her so that he/she is away & $37(41.1)$ & $10(11.1)$ & $43(47.7)$ \\
\hline Community members refuse to associate with a schizophrenic patient & $44(48.9)$ & $17(18.9)$ & $39(32.3)$ \\
\hline Community leisure activities are for normal people, not for schizophrenic patients & $39(43.3)$ & $16(17.8)$ & $35(38.9)$ \\
\hline Respect schizophrenic patients as individuals & $20(22.3)$ & $11(12.2)$ & $59(65.5)$ \\
\hline
\end{tabular}

Table 7 reveals that $(30.3 \%$, and $26.7 \%)$ of the studied schizophrenic patients agreed that resources are not available in the community where schizophrenic patient can work, and community members accept and love schizophrenic patients are main rehabilitation factors of relapse in the community respectively. On the other hand, (36.3\%) of the studied schizophrenic patients disagreed that community members afraid to deal with schizophrenic patient, whereas; $(38.6 \%)$ agreed on that matter.

Table 7. Community factors of relapse as reported by the studied schizophrenic patients $(n=90)$.

\begin{tabular}{|c|c|c|c|}
\hline \multirow{2}{*}{ Rehabilitation in the community factors } & Disagree & Uncertain & Agree \\
\hline & $N(\%)$ & $N(\%)$ & $N(\%)$ \\
\hline Resources are not available in the community where schizophrenic patients can work & $52(57.8)$ & $14(15.6)$ & $24(26.7)$ \\
\hline Schizophrenic patients are not able to comprehend a thing when they are told & $48(53.4)$ & $20(22.2)$ & $22(24.4)$ \\
\hline Schizophrenic patient are not able to learn anything new & $43(47.8)$ & $25(27.8)$ & $22(24.4)$ \\
\hline Community members accept and love schizophrenic patient & $31(34.9)$ & $31(34.8)$ & $27(30.3)$ \\
\hline Community members afraid to deal with schizophrenic patients & $32(36.3)$ & $22(25.0)$ & $34(38.6)$ \\
\hline
\end{tabular}

Table 8. Compliance with medications as reported by studied schizophrenic patients $(n=90)$.

\begin{tabular}{|c|c|c|c|}
\hline \multirow{2}{*}{ Compliance with medications } & Disagree & Uncertain & Agree \\
\hline & $N(\%)$ & $N(\%)$ & $N(\%)$ \\
\hline Do you know that you are a mentally ill patient & $43(47.8)$ & $8(8.9)$ & $39(43.3)$ \\
\hline Do you think you need to take psychotropic agents & $46(51.1)$ & $7(7.8)$ & $37(41.1)$ \\
\hline Did you take your medications after you were discharged from the hospital & $36(40)$ & $17(18.9)$ & $37(41.1)$ \\
\hline Did you ever stop your medications by yourself & $28(31.1)$ & $20(22.2)$ & $42(46.6)$ \\
\hline Do you have any information about your illness or the medications that you take & $12(13.4)$ & $14(15.6)$ & $64(71.1)$ \\
\hline
\end{tabular}


Table 8 indicates that, $(71.1 \%, 46.6 \%$, and $43.3 \%)$ of the studied schizophrenic patient respectively agree that they did not have any information about the illness or medications, never stop medication alone, and have insight about their illness are main compliance factor of relapse. Meanwhile; $(51.1 \%, 47.8 \%$, and $40 \%)$ of the studied schizophrenic patients respectively revealed that they disagree in relation to the need to take psychotropic medications, insight about the illness, and take medication after discharge were the mean compliance factors of relapse.

Table 9 shows that, the medications compliance subscale has the highest mean among all the relapse factors subscales $(15.14 \pm 5.16)$; followed by social stigma subscale $(14.47 \pm$ 4.80 ); while the personal factors of relapse has the lowest mean $(10.73 \pm 4.32)$ and the total relapse mean scores was $(66.34 \pm 13.22)$. However, the personal factors of relapse showed the highest variability among all the subscales $(40.26 \%)$.

Table 9. Mean and standard deviation of total relapse scores and subscores among the studied schizophrenic patients ( $n=90)$.

\begin{tabular}{|c|c|c|c|c|c|}
\hline & Mean & SD & Minimum & Maximum & $\mathrm{COV}$ \\
\hline Personal factors & 10.73 & 4.32 & 5.00 & 23.00 & $40.26 \%$ \\
\hline Social factors & 13.38 & 3.49 & 5.00 & 20.00 & $26.08 \%$ \\
\hline Social stigma & 14.47 & 4.80 & 5.00 & 24.00 & $33.17 \%$ \\
\hline Community factors & 13.13 & 4.83 & 5.00 & 25.00 & $36.78 \%$ \\
\hline Medications compliance & 15.14 & 5.16 & 5.00 & 25.00 & $34.08 \%$ \\
\hline Total relapse & 66.33 & 13.23 & 41.00 & 96.00 & $19.94 \%$ \\
\hline
\end{tabular}

Table 10 reveals that, there was a highly statistically significant inverse relationship between patient's income and total medications compliance subscale as $(\mathrm{r}=-.329, \mathrm{p}=.002)$. Also, there was a statistically significant inverse relationship between patient's number of follow-up and total medications compliance subscale as $(\mathrm{r}=-.292, \mathrm{p}=.042)$. Moreover, there was a statistically significant relationship between patient's education and total community factors subscale as $(r=-.259$, $\mathrm{p}=.016$ ). However, there were no statistically significant among the other total relapse scores and subscores and patients' socio-demographic and medical characteristics.

Table 10. Correlation matrix among total relapse scores and subscores and patients' socio-demographic and medical characteristics $(n=90)$.

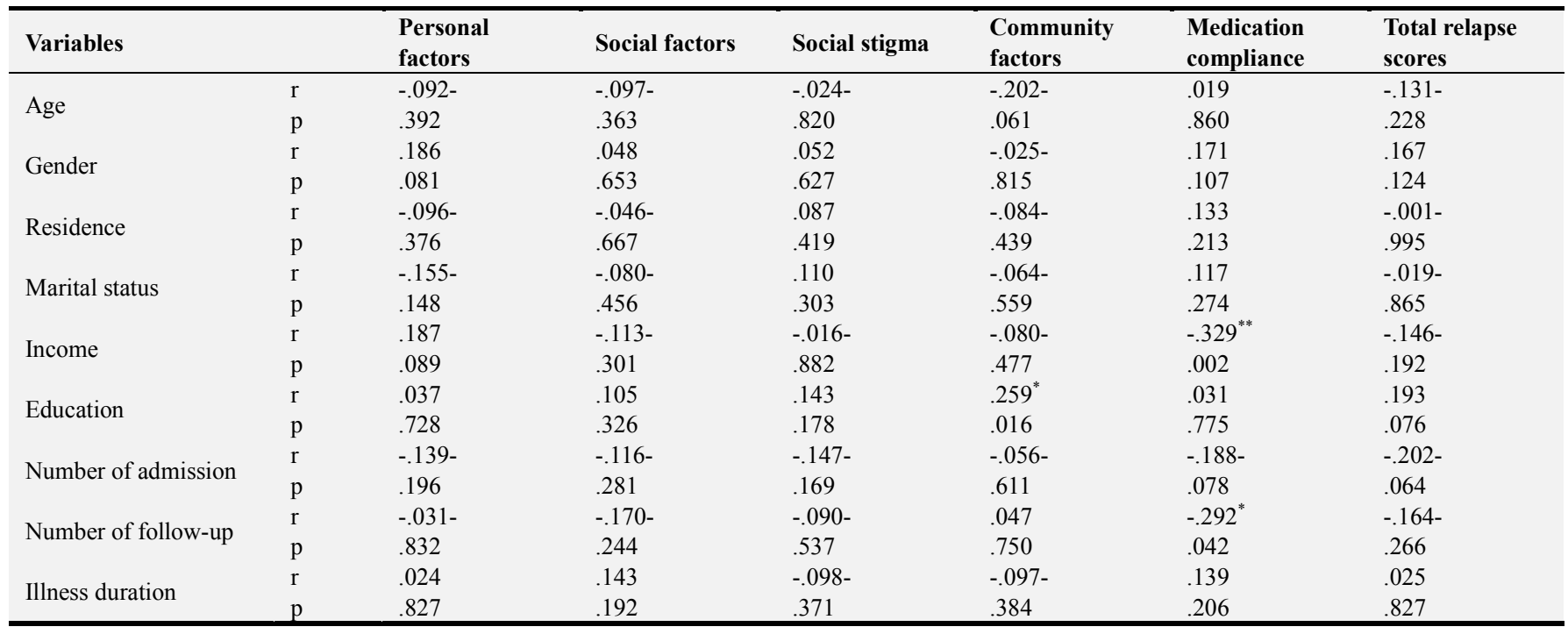

**. Correlation is significant at the 0.01 level (2-tailed)

*. Correlation is significant at the 0.05 level (2-tailed).

\section{Discussion}

The current study results revealed that, the majority of patients were male. The result shows the number of male more than female this could be due to the nature of the society and stigma that discourage the females to visit the community mental health clinic and hospital. Male preponderance was also seen by other studies [5-6 \& 8-9].

Slightly more than one third of the sample was in the age of (25-35) years. This age indicates that schizophrenia affects the productivity and QOL of individuals. This could be due to schizophrenia as a chronic disability illness in which most patients experience frequent periods of psychotic exacerbation and relapse. Relapse in schizophrenia can be associated with progressive functional deterioration, declining treatment response, worsen clinical outcome and escalating caregiver burden. This finding is congruent with Chabungbam, Avashti, and Sharan [8] and Alsherif and Abd Elrahman, [9] who reported the mean age of their sample was around thirty three years old for the relapsed group and around thirty four years old for the readmitted group.

As regards the studied sample subjects' residence, more 
than half of them were from urban areas in Egypt and that was consistent with Chabungbam, Avashti, and Sharan [9]; and Chaurotia, Verma, and Baniya [10]. This result may be due to the increased mental illness stigma in rural areas in Egypt that might affect the patient help seeking behaviors who then become hesitant to visit any psychiatric facility.

Concerning the current sample education, the study showed that almost all the studied patients can read and write and that is incongruent with the results of Chabungbam, Avashti, and Sharan, [8] who reported that almost three thirds of the sample was literate. However, the finding was inconsistent with Chaurotia, Verma, and Baniya [10] who found that $24 \%$ of the relapsed group and $32 \%$ of remitted group were illiterate.

Regarding the marital status of the studied sample shows that slightly more than half of the sample was single. This finding is on the same line of the study by San et al., [5] who found that majority of patients $73.3 \%$ were unmarried and $12.2 \%$ divorced. On the other hand, this result is inconsistent with Abdel-Baki et al., [11], who found that the majority of his studied sample was married. This finding may be related to the stigmatizing nature of the mental illness that prevent most of the schizophrenic patients in the Egyptian society to be married as they considered less than normal people.

Slightly more than half of the sample reported that they have been working as employees or in free jobs and that helped them to have enough income to meet their needs. This result may indicate that when patients have a job to earn income they become independent. This boosts their selfesteem and helps them for that they can contribute something to the family or society. This result may be due to the availability jobs that do not require specific skills in the Egyptian society such as working as carpenter, butcher, farmer and preparing food.

Less than half of the sample was accompanied by their parents during the follow-up in the psychiatric outpatient clinic. This result may be to the fact that half of the sample is single which means that they still live with their parents. The current study showed that three quarters of the studied sample were hospitalized less than three times per year. This finding is incongruent with the results of Chaurotia, Verma, and Baniya [10], who found that most of the psychiatric patients follow-up with a psychiatrist are regularly readmitted to psychiatric facilities each year. According to the researchers' clinical experience and patients perspectives, medication compliance is most buffering factor that usually leads to decreased times of re-hospitalization of the psychiatric patients.

The current study revealed that, communicate effectively and lack of good manner of approach when talking to others were the main factors of relapse among schizophrenic patients. This finding was in the same line with Moritz and Woodward, [12]; and Chabungbam, Avashti, and Sharan, [8]; and Chaurotia, Verma, and Baniya [10]. This finding may be due to mental illness is usually result in impaired communication and consequently lead to impaired social interaction. This finding was also supported by Huxley and
Thornicroft,, [13] pointed out that the nature of mental illness, health problems and discrimination against them could cause interpersonal relationships to deteriorate, leading to reduced social contacts. They are unlikely to have any close friends and many may have no one to turn to for help.

Concerning the relapse social related factors, the study showed that three quarters of the sample disagreed that mentally ill people are dangerous and should not be left alone. For mental illness attacks the self-concept of patients. The level of responsiveness from community members, family members, friends and supportive others such as (peers/professionals) when patients need help also has an impact. This results could be due to that the degree of stigmatization that the community holds This fining is consistent with Chaurotia, Verma, and Baniya [10] and Sagduyu, et al., [14] who reported that $72.2 \%$ of the sample held the opinion that people with schizophrenia are dangerous and $91.8 \%$ believed that these patients could not take responsibility for their own lives. Also, the sample of the current study reported that they do not go to the hospital due to distance and the cost of taking the traffic. This result is similar to the finding of Chaurotia, Verma, and Baniya [10] who reported that that distance would make the patients fail to go for follow up care especially for those living far from the hospital.

Regarding the social stigma factors, the study revealed that less than half of the sample agreed that mentally ill patients do not social contacts and do not engage in social activities that may be due to the stigmatizing nature of their illness. This result could be due to that stigma remains the main obstacle to a better life for people suffering from mental disorders. People diagnosed with schizophrenia still encounter prejudice, difficulties and feel excluded from society. Emsley, Chiliza, Asmal, and Brain [15] "found in their study of stigma by association and psychological factors in relatives of people with mental illness, that $83 \%$ of relatives are burdened by one of the psychological factors; $72 \%$ of women thought the sick relative would have been better off dead; $33 \%$ wished that the patient or they themselves had never been born; spouses did not believe that the patient would be better off dead; $51 \%$ of relatives and $47 \%$ of the spouses reported that the patient's mental illness had Affected them negatively and they could not have company of their own".

Concerning the medication adherence, the study reported that half of the sample disagreed to take their psychotropic medications and they stop taking these medications by themselves. This result may be due to that one of the major reasons for relapse is failure to follow the medication regimen. Discounting medications almost certainly leads to a relapse and may be actually be a stressor that cause severe and rapid relapse. Moreover, unpleasant side effects of medication, social adversity and isolation also play a role. This finding is similar to Chaurotia, Verma, \& Baniya [10] and Beebe, [16] who pointed out that psychiatric medication can result in unpleasant adverse effects such as weight gain, risk of heart disease, vascular disease, and diabetes cited as 
some factors that led to psychiatric patients' non-adherence to treatment. The risk of non-adherence leads to a risk of relapse.

The study revealed no significant association was found between the patient's socio-demographic and medical data and the relapse related factors. In contrast to Chaurotia, Verma, and Baniya [10] who reported that male are more likely to be relapsed than females with schizophrenia. Also, the fining is incongruent with Chabungbam, Avashti, and Sharan, [8] who found that unemployment is strong predictor of relapse among schizophrenic patients. However, as it was noted half of the sample were working and as it was reported by Chaurotia, Verma, and Baniya [10] employment improves socioeconomic status, provides social interaction, and helps provide meaning or purpose to an individual and can be viewed as therapeutic.

\section{Conclusion}

The study concluded that many factors can interfere with the recovery process of schizophrenic patients such as, lack of social and community support, non compliance to medication, and stigma. However, patient's sociodemographic, and medical data might not be indicators for relapse among schizophrenic patients.

\section{Recommendations}

1. Further studies are needed to investigate these reported factors on larger sample size.

2. Other factors should be consider in further research investigation such as, the stress factors and caregivers perspectives compared to the patients perspectives.

3. Monitoring for prodromal symptoms of relapse by treatment providers, patients, family members, and others in frequent contact with the patient.

4. Further emphasis should be focused on training of mental health nurses who will provide quality mental health services for in-patient and outpatient mentally ill patients.

5. Primary mental health care must be enhanced to prevent relapse among mentally ill patients.

\section{References}

[1] Brink, C., Van der Walt, C. and Van Rensburg, G. (2012). Fundamentals of Research Methodology for Health Care Professionals. $3^{\text {rd }}$ ed. Lansdown: Juta and Company Ltd.

[2] Kane J. M., (2007)."Treatment strategies to prevent relapse and encourage remission," J Clin Psychiatry, vol. 68 Suppl 14, pp. 27-30.
[3] Harris M. G., Henry, L. P. Harrigan, S. M. Purcell, R., O. S. Schwartz, S. E. Farrelly, A. L. Prosser, H. J. Jackson and P. D. McGorry, (2005). "The relationship between duration of untreated psychosis and outcome: an eight-year prospective study," Schizophrenia Research, vol. 79, no. 1, pp. 85-93.

[4] American Psychiatric, A. American Psychiatric A. and D.-I. (2016). Task Force on, Diagnostic and statistical manual of mental disorders: DSM-IV-TR, American Psychiatric Association, Washington, DC.

[5] San, L. Bernardo, M. Gomez A., and Pena, M.(2013). Factors associated with relapse in patients with schizophrenia," Int J Psychiatry Clin Pract, vol. 17, no. 1, pp. 2-9.

[6] Sariah, A. E. Outwater A. H. and Malima, K. I.(2014)."Risk and protective factors for relapse among individuals with schizophrenia: a qualitative study in Dar es Salaam, Tanzania," BMC Psychiatry, vol. 14, pp. 240,.

[7] Burns, N. and Grove, S. K. (2011). Understanding Nursing Research. 5th ed. Maryland Heights: Elsevier.

[8] Chabungbam, G. Avasthi A. and Sharan, P.(2007)."Sociodemographic and clinical factors associated with relapse in schizophrenia," Journal of Psychiatry Clinical Neuroscience, vol. 61, no. 6 , pp. 587-593.

[9] Alsherif, Z. and Abd Elrahman, S.(2013). Schizophrenic Patients's Perception of Factors Leading to Relapse. Journal of American Science; 9(12), PP512-517.

[10] Chaurotia, V., Verma, K., and Baniya, B. (2016). A Study of Psychosocial Factor Related with Relapse in Schizophrenia. IOSR-JDMS, Volume 2016; 15(4): 26-34.

[11] Abdel-Baki, A. Lesage, A. Nicole, L. Cossette, M. Salvat E., and Lalonde, P.(2011)."Schizophrenia, an illness with bad outcome: myth or reality?," Canadian Journal of Psychiatry, vol. 56, no. 2, pp. 92-101.

[12] Moritz S., and Woodward, T. S.(2005). "Jumping to conclusions in delusional and non-delusional schizophrenic patients," Br J Clin Psychol, vol. 44, no. Pt 2, pp. 193-207.

[13] Huxley P. and Thornicroft, G.(2003). "Social inclusion, social quality and mental illness," Br J Psychiatry, vol. 182, pp. 289290.

[14] Sagduyu, A. Aker, T. Ozmen, E. Uguz S., Ogel K. and Tamar, D. (2003). "[Relatives' beliefs and attitudes towards schizophrenia: an epidemiological investigation]," Turk Psikiyatri Derg, vol. 14, no. 3, pp. 203-212.

[15] Emsley, R., Chiliza, B., Asmal, L. and Brain, H.(2013). The nature of relapse in schizophrenia, BMC Psychiatry, 13: 50 .

[16] Beebe, L. H. (2002). "Problems in community living identified by people with schizophrenia," J Psychosocial Nursing Mental Health Services, vol. 40, no. 2, pp. 38-45. 